\title{
PUFA status at birth and allergy-related phenotypes in childhood: a pooled analysis of the Maastricht Essential Fatty Acid Birth (MEFAB) and RHEA birth cohorts
}

\author{
Nikos Stratakis ${ }^{1,2 *}$, Marij Gielen ${ }^{1}$, Katerina Margetaki ${ }^{2}$, Renate H. M. de Groot ${ }^{1,3}$, Maria Apostolaki ${ }^{4}$, \\ Georgia Chalkiadaki ${ }^{2}$, Marina Vafeiadi ${ }^{2}$, Vasiliki Leventakou ${ }^{2}$, Roger W. Godschalk ${ }^{5}$, \\ Manolis Kogevinas $^{6,7,8}$, Euripides G. Stephanou ${ }^{4,9}$, Maurice P. Zeegers ${ }^{1,10_{\dagger}}$ and Leda Chatzi ${ }^{1,2} \dagger$ \\ ${ }^{1}$ Section of Complex Genetics and Epidemiology, Department of Genetics and Cell Biology, School of Nutrition and Translational \\ Research in Metabolism, Faculty of Health, Medicine and Life Sciences, Maastricht University, 6200 MD Maastricht, The Netherlands \\ ${ }^{2}$ Department of Social Medicine, Faculty of Medicine, University of Crete, 71003 Heraklion, Greece \\ ${ }^{3}$ Welten Institute - Research Centre for Learning, Teaching and Technology, Open University of the Netherlands, \\ 6419 AT Heerlen, The Netherlands \\ ${ }^{4}$ Environmental Chemical Processes Laboratory (ECPL), Department of Chemistry, University of Crete, 71 o03 Heraklion, Greece \\ ${ }^{5}$ Department of Pharmacology \& Toxicology, School of Nutrition and Translational Research in Metabolism (NUTRIM), \\ Faculty of Health, Medicine and Life Sciences, Maastricht University, 6200 MD Maastricht, The Netherlands \\ ${ }^{6}$ ISGlobal, Centre for Research in Environmental Epidemiology (CREAL), 08003 Barcelona, Spain \\ ${ }^{7}$ IMIM Hospital del Mar Medicine Research Institute, 08003 Barcelona, Spain \\ ${ }^{8}$ Spanish Consortium for Research in epidemiology and Public Health (CIBERESP), 28029 Madrid, Spain \\ ${ }^{9}$ The Cyprus Institute, 2121 Nicosia, Cyprus \\ ${ }^{10}$ School CAPHRI: Care and Public Health Research Institute, Maastricht University, 6200 MD Maastricht, The Netherlands
}

(Submitted 28 March 2017 - Final revision received 6 September 2017 - Accepted 7 September 2017)

\section{Abstract}

Lower prenatal exposure to $n$-3 PUFA relative to $n$ - 6 PUFA has been hypothesised to influence allergy development, but evidence remains largely inconsistent. In the Dutch Maastricht Essential Fatty Acid Birth (MEFAB) ( $n$ 293) and Greek RHEA Mother-Child ( $n$ 213) cohorts, we investigated whether cord blood phospholipid PUFA concentrations are associated with symptoms of wheeze, asthma, rhinitis and eczema at the age of 6-7 years. Information on allergy-related phenotypes was collected using validated questionnaires. We estimated relative risks (RR) and $95 \% \mathrm{CI}$ for associations of PUFA with child outcomes using multivariable generalised linear regression models. In pooled analyses, higher concentration of the $n-3$ long-chain EPA and DHA and a higher total $n-3: n-6$ PUFA ratio were associated with lower risk of current wheeze (RR 0.61; $95 \%$ CI 0.45, 0.82 per SD increase in EPA + DHA and 0.54; 95\% CI 0.39, 0.75 per unit increase in the $n$-3:n-6 ratio) and reduced asthma risk (RR 0.50; $95 \% \mathrm{CI} 0 \cdot 31,0.79$ for EPA + DHA and 0.43; $95 \% \mathrm{CI} 0 \cdot 26,0 \cdot 70$ for the $n-3: n-6$ ratio). No associations were observed for other allergy-related phenotypes. The results were similar across cohorts. In conclusion, higher EPA and DHA concentrations and a higher $n$-3: $n-6$ fatty acid ratio at birth were associated with lower risk of child wheeze and asthma. Our findings suggest that dietary interventions resulting in a marked increase in the $n-3: n-6$ PUFA ratio, and mainly in $n$ - 3 long-chain PUFA intake in late gestation, may reduce the risk of asthma symptoms in mid-childhood.

Key words: PUFA: Cord blood: Asthma: Eczema: Rhinitis: Childhood

Childhood asthma and other allergy-related diseases pose a substantial health burden in developed countries ${ }^{(1)}$. Fetal life is a critical time period of susceptibility during which a nutritional stressor can affect the development and maturation of respiratory and immune systems, and thus have irreversible influences on the development of allergy $^{(2)}$. In this context, it has been hypothesised that a lower intake of $n-3$ relative to $n-6$ PUFA might play a role in the aetiology of allergy-related diseases ${ }^{(3)}$. PUFA of $n-6$ and $n-3$ families can be readily transferred across the placenta ${ }^{(4)}$, and have been shown in animal and in vitro studies to exert immunomodulatory effects ${ }^{(5,6)}$. Specifically, $n-6$ PUFA have been suggested to promote inflammation and

Abbreviations: FAME, fatty acid methyl ester; MEFAB, Maastricht Essential Fatty Acid Birth; RR, relative risk.

* Corresponding author: N. Stratakis, email n.stratakis@maastrichtuniversity.nl

$\dagger$ These authors contributed equally and share senior authorship. 
increase allergic-disease activity, whereas $n-3$ PUFA seem to have an opposite effect ${ }^{(5,6)}$

However, reports from human studies do not conclusively support that an increase in $n-3$ PUFA status, a decrease in $n-6$ PUFA status or both in the prenatal period is an effective strategy to prevent allergy-related symptoms in childhood. A 2016 systematic review and meta-analysis of prospective cohort studies assessing maternal fish or $n$ - 3 long-chain PUFA intake during pregnancy and of trials assessing fish-oil supplementation reported that the hypothesis linking maternal $n$ - 3 PUFA intake to childhood allergic disease cannot unequivocally be confirmed or rejected $^{(7)}$. Two subsequent trials on fish-oil supplementation also showed discrepant results, with reports of null ${ }^{(8)}$ or beneficial (i.e. lower incidence) effects $^{(9)}$. Similarly, findings from studies assessing prenatal PUFA biomarker concentrations, which constitute accurate surrogates of fetal exposure ${ }^{(10)}$, have produced mixed results. The British Southampton Women's Survey and Avon Longitudinal Study of Parents and Children (ALSPAC) studies reported lower rates of childhood wheeze symptoms with higher prenatal $n$-3 PUFA concentration ${ }^{(11)}$ or a lower $n-6: n-3$ fatty acid ratio ${ }^{(12)}$. In contrast, the Dutch Generation R study showed maternal concentration of $n-6$ PUFA, but not of $n-3$ PUFA levels, to be associated with decreased asthma risk in childhood ${ }^{(13)}$, whereas other studies in the Netherlands, Germany and Singapore found no associations between any PUFA and child asthma or wheeze symptoms ${ }^{(14-16)}$. A lower risk of childhood eczema was shown with increasing cord blood $n$-3 PUFA concentration in the Spanish INMA cohort ${ }^{(17)}$. The Generation $\mathrm{R}$ found a higher eczema risk with higher maternal $n$-6 PUFA concentration ${ }^{(13)}$, whereas the Dutch KOALA study reported a lower risk with increasing $n-6: n-3$ fatty acid ratio ${ }^{(14)}$. Other studies showed weak or null associations of prenatal PUFA concentrations with rates of childhood eczema ${ }^{(12,15,16)}$. Only a few studies have specifically examined childhood rhinitis and found no associations with prenatal PUFA concentrations ${ }^{(14-16)}$.

Contradictory findings of prior studies may at least partly be explained by heterogeneity in exposure and health outcome definitions, and differences in adjustment for confounding variables. Moreover, most of the trials were prone to methodologic limitations, such as selective reporting and absence of blinding of participants or personnel. Given the substantial health burden of allergic diseases, it is important to better characterise the potential role that PUFA may play in the development of asthma and other allergy-related diseases. Pooled analyses of diverse populations constitute an important step towards more generalisable conclusions. Hence, in the present study, we harmonised and pooled individual data of two cohorts from West and South Europe to assess whether $n-3$ and n-6 PUFA concentrations in cord blood phospholipids, reflecting fetal exposure in late pregnancy, are associated with symptoms of wheeze, asthma, rhinitis and eczema in children aged 6-7 years.

\section{Methods}

\section{Study population}

The Maastricht Essential Fatty Acid Birth (MEFAB, www. mefab.org) cohort $^{(18)}$ enrolled 1334 pregnant women at the time of their first antenatal visit in three maternity clinics in the province of Limburg, the Netherlands, between 1989 and 1995. Inclusion criteria for participation in the study were as follows: gestational age $<16$ weeks, diastolic blood pressure $<90 \mathrm{mmHg}$ and no cardiovascular, neurological, renal or metabolic disorder. A total of 1203 women with singleton pregnancies were followed up until delivery, and 1008 them provided umbilical cord blood samples. Between 1997 and 2000, when children were 7 years of age (mean age $7 \cdot 3$ (sD 0.3 ) years), we conducted a follow-up evaluation; eligibility criteria included childbirth date before 1994, singleton pregnancy and availability of cord blood fatty acid measurements. Among 750 eligible children, 306 participated in the follow-up study, during which information on allergy-disease symptoms was obtained for 293 of them. A flow chart of the MEFAB participants is shown in online supplementary Fig. S1.

The RHEA Mother-Child Study in Crete (Greece, www. rhea.gr) ${ }^{(19)}$ enrolled pregnant women during 2007-2008 at the time of the first major ultrasound examination at 10-13 weeks of gestation if they were resident at the prefecture of Heraklion, $>16$ years of age and without communication handicaps. During the study period, 1610 pregnant women agreed to participate and 1363 singleton pregnancies were followed up until delivery. A random subgroup of 500 umbilical cord blood samples was essayed for fatty acid levels in RHEA. For the child's follow-up, the mothers were contacted at 9 months ( $n$ 1223), 4 years ( $n$ 873) and 6 years of age ( $n$ 608); sample sizes correspond to singleton children. In the present analysis, we included 228 children aged 9 months (mean age 9.1 (SD 1.9) months), 222 aged 4 years $(4 \cdot 2$ (sD $0 \cdot 2)$ years) and 213 aged 6 years (6.6 (SD 0.3) years) with available information on fatty acid exposure and allergy-related symptoms. A flow chart of the RHEA participants is shown in the online Supplementary Fig. S2.

In both cohorts, participants' parents or legal guardians provided written informed consent. The study was conducted according to the guidelines laid down in the Declaration of Helsinki. All procedures involving human subjects were approved by the local authorised institutional review boards (Medical Ethics Committee of the University Hospital Maastricht and the University of Maastricht, the Netherlands, for the MEFAB cohort, and the review board of the University Hospital in Heraklion, Crete, Greece for the RHEA cohort).

\section{PUFA analysis}

In the MEFAB birth cohort, blood samples from the umbilical vein of infants were collected immediately after delivery in EDTA-containing tubes. Plasma was separated from blood cells by centrifugation and stored in aliquots at $-80^{\circ} \mathrm{C}$ until analysis. Methods of fatty acid analysis and the phospholipid PUFA identified in MEFAB have been described in detail elsewhere ${ }^{(20)}$.

In the RHEA birth cohort, umbilical cord blood samples were collected at delivery in 10-ml BD gel separator vacutainers. Serum was separated within $2 \mathrm{~h}$ after collection using centrifugation and stored in $0.5-\mathrm{ml}$ aliquots at $-80^{\circ} \mathrm{C}$ until assayed. The analysis of phospholipid fatty acids, including PUFA, was conducted in the Environmental Chemical Processes Laboratory (University of Crete, Greece) following a standard methodology, 
as described by Glaser $e t a l .{ }^{(21)}$. In brief, a fatty acid methyl ester (FAME) sample was obtained using direct in situ transesterification. The individual FAME were quantified by GC with flame ionisation detection. GC analysis was carried out on a Shimadzu2010 chromatograph equipped with AOC-20i auto-injector (Shimadzu Europe). Separation of serum phospholipid FAME was performed on a SP-2560-fused silica capillary column ( $100 \mathrm{~m} \times 0.25 \mathrm{~mm}$ internal diameter, $0.2 \mu \mathrm{m}$ film thickness; Supelco) using $\mathrm{He}$ as the carrier gas at a constant flow of $1.1 \mathrm{ml} / \mathrm{min}$. The injector port was maintained at $250^{\circ} \mathrm{C}$ and $1.6 \mu \mathrm{l}$ sample volumes were injected at a split ratio of 1:20. The GC oven initial temperature was $130^{\circ} \mathrm{C}$, and then increased at a rate of $4^{\circ} \mathrm{C} / \mathrm{min}$ to $170^{\circ} \mathrm{C}$ and then at a rate of $2^{\circ} \mathrm{C} / \mathrm{min}$ to $210^{\circ} \mathrm{C}$ and retained for $20 \mathrm{~min}$. Detector temperature was kept at $260^{\circ} \mathrm{C}$. Individual FAME peaks were identified by comparison of their retention times with SUPELCO 37 Component FAME mix (Supelco).

PUFA composition of plasma phospholipids is similar to that of serum phospholipids, and slight differences in the analytical methods used for fatty acid analysis in serum and plasma do not appear to affect the way that PUFA composition can be interpreted $^{(22,23)}$.

For the present analysis, our primary exposures of interest were the dietary essential $n$-3 PUFA precursor, $\alpha$-linolenic acid (ALA, 18:3n-3), the sum of its major biologically active metabolic products EPA (20:5n-3) and DHA (DHA, 22:6 $n-3)^{(24)}$, and the sum of total $n-3$ fatty acids assayed in each cohort; the dietary essential $n-6$ PUFA precursor, linoleic acid (LA, 18:2n-6), its major metabolic product arachidonic acid (AA, $20: 4 n-6)^{(25)}$ and the sum of total $n-6$ fatty acids assayed; and the total $n-3: n-6$ PUFA ratio.

Secondary exposures of interest were intermediate metabolic products in the biosynthetic pathway of PUFA that have been previously associated with pregnancy and child allergyrelated outcomes including the $n-3$ docosapentaenoic acid (DPA, 22:5n-3), the $n$-6 $\gamma$-linolenic acid (GLA, 18:3n-6), dihomo- $\gamma$-linolenic acid (DGLA, 20:3n-6) and osbond acid $(22: 5 n-6)^{(13,26,27)}$. Individual fatty acid measurements were expressed as weight percentage of total fatty acids present in the chromatogram (wt\%).

\section{Childhood allergy-related outcomes}

In both cohorts, information on wheeze, asthma, rhinitis and eczema occurrence was obtained by questionnaires adapted from the International Study on Asthma and Allergy in Childhood (ISAAC) ${ }^{(28)}$. We defined current wheeze as the presence of any episode of wheezing or whistling in the chest in the past 12 months. Asthma ever was defined as ever-reported doctor diagnosis of asthma, whereas current asthma was defined as a positive answer to both ever-reported diagnosis of asthma and presence of wheezing or whistling in the chest in the past 12 months $^{(29)}$. We defined current rhinitis as the presence of sneezing or a runny or blocked nose in the past 12 months without common cold or flu ${ }^{(30)}$. We defined current eczema as the presence of an itchy rash in the past 12 months that affected any of the following places: the folds of the elbows, behind the knees, in front of the ankles, under the buttocks or around the neck, ears or eyes ${ }^{(31)}$. For the MEFAB cohort, we used available information on allergy-related outcomes at the age of 7 years. For the RHEA cohort, we used information on wheeze symptoms at three time points (at 9 months, 4 years and 6 years), and information on asthma, rhinitis and eczema at 6 years. Although in RHEA there was available information on asthma, rhinitis and eczema at 4 years of age, we did not include these outcomes in the current analysis owing to very small numbers.

\section{Covariates}

We defined important confounders or effect modifiers as similarly as possible among the cohorts. Information on maternal weight and height at study entry, which were used to calculate maternal BMI $\left(\mathrm{kg} / \mathrm{m}^{2}\right)$, gestational weight gain from study entry until birth (kg), maternal smoking during pregnancy (yes/no), maternal age at delivery (in years), delivery type (vaginal delivery or caesarean section), parity (primiparous or multiparous), birth weight (g), gestational age (weeks) and child sex (male or female) was collected by means of self-administered questionnaires or hospital records. Information on breastfeeding (months), number of siblings (0, 1, 2, or $\geq 3$ ), parental education (cohort-specific definitions of low, medium or high according to the highest completed education level of either parent) and history of parent atopy (presence of asthma, rhinitis or eczema to either one or both parents; yes/no) was obtained through self-administered questionnaires. At 6-7 years of age, child weight and height were measured according to standard procedures in both cohorts, and BMI $\left(\mathrm{kg} / \mathrm{m}^{2}\right)$ was calculated. In RHEA, we had available information on Mediterranean diet adherence in pregnancy calculated with an a priori defined score using a validated $\mathrm{FFQ}^{(32)}$. In MEFAB, we also collected fasting child blood samples at the age of outcome assessment and measured plasma levels of PUFA with standard procedures as described previously ${ }^{(33)}$.

\section{Statistical analysis}

Characteristics of the study population were determined using descriptive analysis. Differences in the baseline characteristics between children included the present analysis and those excluded were studied using Pearson's $\chi^{2}$ test for categorical variables and Student's $t$ test for continuous ones.

We calculated standardised $z$ scores for concentrations of all PUFA in each cohort to enable the comparison and pooling of cohort-specific effect estimates. We used multivariable generalised linear models for binary outcomes (with log link, Poisson distribution and robust variance estimator) ${ }^{(34)}$ to estimate relative risks (RR) and $95 \% \mathrm{CI}$ for the associations of each PUFA exposure with the outcomes of interest. We detected no departures from linearity in the exposure-outcome associations using a Wald test with the STATA command nlcheck ${ }^{(35)}$. We conducted cohort-specific analyses. We also performed pooled analyses by including a cohort indicator variable in the models and tested for heterogeneity in the associations between the two cohorts by examining the interaction between PUFA concentrations and cohort.

Following a Directed Acyclic Graph approach (online Supplementary Fig. S3), we selected the following variables for 
model adjustment: maternal age, maternal BMI at study entry, gestational weight gain, maternal smoking during pregnancy, parental education, parity, parent atopy, child sex and age at outcome assessment. We did not include birth weight or gestational age in our main models, as they might be in the intermediate pathway between prenatal PUFA levels and child allergy-related outcomes.

To further assess our research hypothesis, we conducted additional analyses using different levels of available information within each cohort. First, we assessed the extent to which child plasma PUFA levels measured at the age of outcome assessment could explain the observed associations by further adjusting for them in the models for MEFAB and evaluating whether they changed the effect estimates. Second, we conducted further adjustment for Mediterranean diet adherence in pregnancy in the RHEA models. Third, we examined associations between the primary PUFA of interest and current wheeze symptoms at 9 months and 4 years of age in the RHEA birth cohort.

To increase sample size (precision) and reduce bias due to missing data, we imputed missing covariate data. Using multiple imputations with chained equations (MICE), we generated fifty imputed data sets for each cohort ${ }^{(36,37)}$. The imputation model included exposures, outcomes, confounders, as well as additional auxiliary variables such as maternal origin, mode of delivery, pregnancy outcomes, breast-feeding duration, sibship size and child BMI. In analytic models, we combined estimates from the imputed data sets with the use of Rubin's rules ${ }^{(36)}$

Sensitivity analyses were performed to assess the robustness of our results. We repeated the analyses by including only those participants with no missing covariate data (complete case analysis). We made further adjustment for BMI at the age of outcome assessment to assess the extent to which child adiposity could influence the observed associations. We also repeated the analyses after including birth weight and gestational age in the multivariable models. Finally, we examined effect measure modification by child sex (boy $v$. girl) ${ }^{(38)}$ and parent atopy (yes $v$. no $)^{(39,40)}$ in the associations of the total $n-3: n-6$ PUFA ratio with child outcomes by assessing both multiplicative and additive interaction measures between the potential effect modifier (one at a time) and the exposure ${ }^{(41,42)}$. Statistical significance for effect modification was defined as $P<0 \cdot 10$.

Our study including 506 participants had $80 \%$ power at a $5 \%$ significance level to detect an effect size of as small as $48 \%$ for risk estimates of current asthma (the outcome with the lowest prevalence in the pooled data set; $4.6 \%$ ) per SD increase in fatty acid concentrations, which is similar to those reported in previous studies ${ }^{(13-15)}$. Power analysis was conducted using GPOwer version $3.1^{(43)}$. All other analyses were conducted with STATA version 13.0 (StataCorp).

\section{Results \\ Participant characteristics}

In the pooled data set, mothers had a mean age at delivery of $29 \cdot 8$ (SD 4.5) years. Of all parents included in this analysis, 37\% had a high education level. The total ratio of boys to girls was 1.29. Participants' characteristics of the separate cohorts are

presented in Table 1. In non-response analysis, we saw that, compared with children without follow-up measurements, those with follow-up data had mothers who were more likely to be older at delivery and multiparous in MEFAB, whereas in

Table 1. Parental and child characteristics in Maastricht Essential Fatty Acid Birth (MEFAB) and RHEA Mother-Child birth cohorts (Mean values and standard deviations; numbers and percentages)

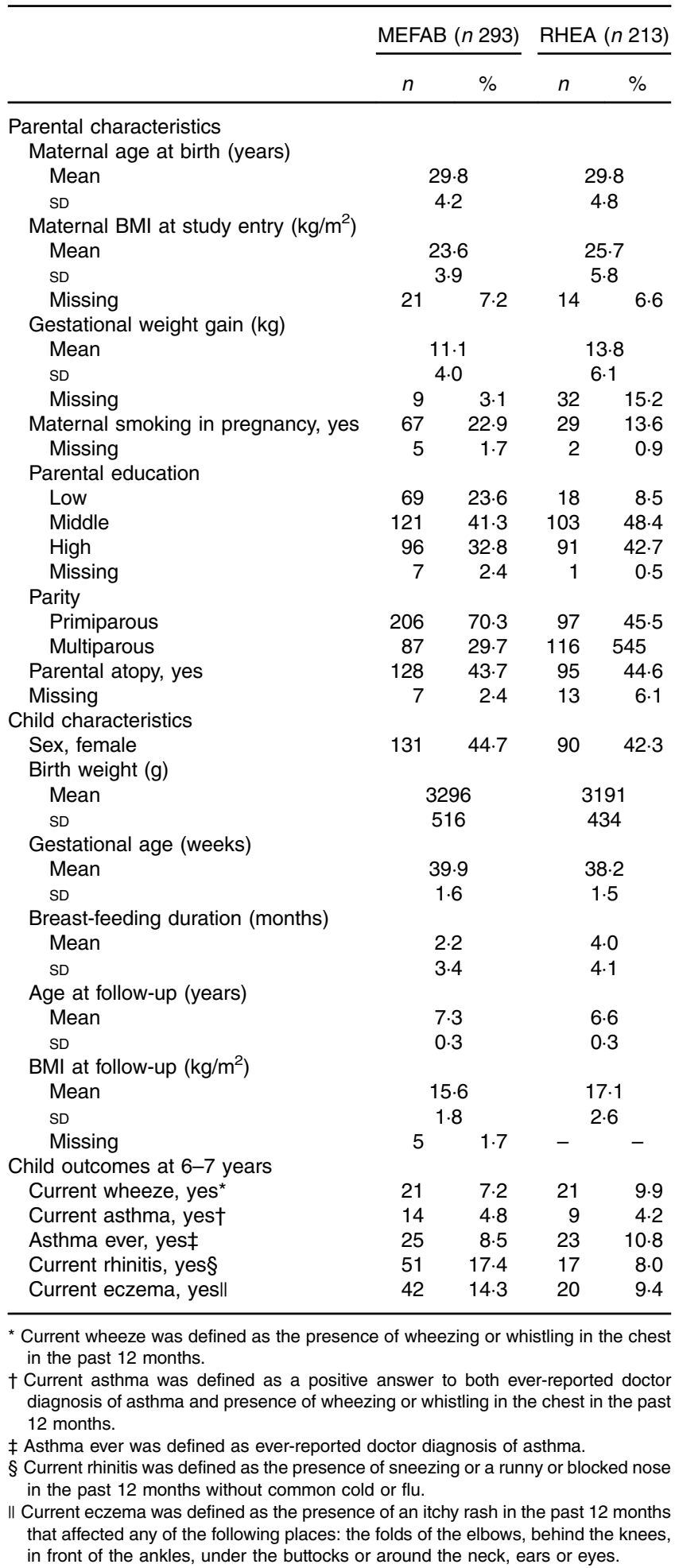


RHEA children with follow-up data were more likely to be male (online Supplementary Table S1).

Table 2 shows the distribution of the cord blood phospholipid PUFA measured in each cohort. Mean concentrations of $n-3$ and $n$-6 PUFA in MEFAB were 6.89 (SD 1.59) and 32.16 (SD 1.69) $\mathrm{wt} \%$, respectively; the corresponding values for RHEA were 5.38 (sD 1.12) and 32.06 (sD 2.35) wt\%.

\section{Primary/pooled analyses}

Interaction $P$ values between PUFA concentrations and cohort were indicative of no significant heterogeneity between cohorts for most exposure-outcome associations, although the magnitude and precision of some effect estimates differed slightly (Table 3 and online Supplementary Tables S2 and S3).

Table 2. Distribution of cord blood phospholipid PUFA levels in Maastricht Essential Fatty Acid Birth (MEFAB) and RHEA Mother-Child birth cohorts (Mean values and standard deviations)

\begin{tabular}{|c|c|c|c|c|}
\hline & \multicolumn{2}{|c|}{ MEFAB $(n$ 293) } & \multicolumn{2}{|c|}{ RHEA $(n 213)$} \\
\hline & Mean & SD & Mean & SD \\
\hline Total $n$-3 PUFA (wt\%) & $6 \cdot 89$ & 1.59 & 5.38 & $1 \cdot 12$ \\
\hline ALA (wt\%) & 0.05 & 0.11 & 0.08 & 0.05 \\
\hline DPA (wt\%) & 0.45 & $0 \cdot 17$ & - & - \\
\hline $\mathrm{EPA}+\mathrm{DHA}(\mathrm{wt} \%)$ & $6 \cdot 29$ & 1.44 & $5 \cdot 28$ & $1 \cdot 10$ \\
\hline Total n-6 PUFA (wt\%) & $32 \cdot 16$ & 1.69 & 32.06 & 2.35 \\
\hline LA (wt\%) & $7 \cdot 70$ & 1.30 & $10 \cdot 41$ & 1.95 \\
\hline GLA (wt\%) & 0.05 & 0.04 & 0.37 & 0.11 \\
\hline DGLA (wt\%) & $5 \cdot 14$ & 0.89 & 3.91 & 0.71 \\
\hline AA (wt \%) & $16 \cdot 63$ & 1.60 & $17 \cdot 04$ & 2.42 \\
\hline Osbond acid (wt\%) & 0.83 & 0.27 & - & - \\
\hline Total $n-3: n-6$ PUFA ratio & 0.22 & 0.06 & 0.17 & 0.04 \\
\hline
\end{tabular}

AA, arachidonic acid (20:4n-6); ALA, $\alpha$-linolenic acid (18:3n-3); DGLA, dihomo- $\gamma-$ linolenic acid (20:3n-6); DHA, $22: 6 n-3$; DPA, docosapentaenoic acid $(22: 5 n-3)$; EPA, $20: 5 n-3$; GLA, $\gamma$-linolenic acid $(18: 3 n-6)$; LA, linoleic acid $(18: 2 n-6)$; osbond acid, 22:5n-6; total $n-3(n-6)$ PUFA, the sum of $n-3(n-6)$ PUFA present in the chromatogram; wt $\%$, weight percentage of total fatty acids measured.
In pooled analyses, we found that higher total $n$-3 PUFA concentration was associated with a lower risk of current wheeze, whereas an inverse association was observed for total $n$-6 PUFA (pooled RR 0.60; $95 \%$ CI 0.45, 0.81 and 1.54; $95 \%$ CI $1 \cdot 09,2 \cdot 17$ per sD increase in $n-3$ and $n-6$ PUFA, respectively). Accordingly, a higher ratio of total $n-3: n-6$ fatty acids at birth was associated with a lower risk of current wheeze (pooled RR 0.54; 95\% CI 0.39, 0.75 per unit increase) (Table 3). When we examined current asthma, we found a lower risk with higher n-3 PUFA concentration (pooled RR 0.49; $95 \%$ CI 0.30, 0.75) and a higher $n-3: n-6$ fatty acid ratio (pooled RR $0 \cdot 43 ; 95 \% \mathrm{CI}$ $0.26,0.70$ ) (Table 3). Associations for ever-reported doctor diagnosis of asthma were in the same direction and magnitude (online Supplementary Table S2). No associations with current rhinitis and eczema symptoms at 6-7 years were found for total $n-6$ PUFA and $n-3$ PUFA concentrations, and the $n-3: n-6$ ratio (online Supplementary Table S3).

In individual fatty acid analyses, we found EPA + DHA concentration in cord blood to be associated with decreased risk of both current wheeze and current asthma (pooled RR 0.61; $95 \%$ CI $0.45,0.82$ and $0.50 ; 95 \%$ CI $0.31,0.79$ per SD increase in $\mathrm{EPA}+\mathrm{DHA}$, respectively) (Table 3 ). Similarly, a protective association was observed between EPA +DHA and risk of ever asthma (online Supplementary Table S2). No associations with any of the outcomes examined were found for the $n-3$ ALA, and the $n-6$ LA and AA in the pooled data set (Table 3 and online Supplementary Tables S2 and S3). Results from the crude models adjusted only for age and sex did not materially differ from those of the main adjusted models (online Supplementary Table S4).

\section{Secondary analyses}

In secondary analyses, we found no associations of the $n-3$ DPA and n-6 GLA, DGLA and osbond acid with allergy-related outcomes (online Supplementary Tables S5 and S6). In addition, inclusion of child plasma PUFA concentrations at the age of outcome assessment in MEFAB models did not modify the

Table 3. Associations of cord blood phospholipid PUFA levels with current wheeze and asthma at 6-7 years of age in Maastricht Essential Fatty Acid Birth (MEFAB) and RHEA Mother-Child cohorts, separately and in pooled analysisł

(Relative risks (RR) and $95 \%$ confidence intervals)

\begin{tabular}{|c|c|c|c|c|c|c|c|c|c|c|c|c|}
\hline & \multicolumn{6}{|c|}{ Current wheeze§ } & \multicolumn{6}{|c|}{ Current asthmall } \\
\hline & \multicolumn{2}{|c|}{ MEFAB ( $n$ 293) } & \multicolumn{2}{|c|}{ RHEA ( $n$ 213) } & \multicolumn{2}{|c|}{ Pooled (n 506) } & \multicolumn{2}{|c|}{ MEFAB $(n$ 293) } & \multicolumn{2}{|c|}{ RHEA ( $n$ 213) } & \multicolumn{2}{|c|}{ Pooled ( $n$ 506) } \\
\hline & $\mathrm{RR}$ & $95 \% \mathrm{Cl}$ & $\mathrm{RR}$ & $95 \% \mathrm{Cl}$ & $\mathrm{RR}$ & $95 \% \mathrm{Cl}$ & $\mathrm{RR}$ & $95 \% \mathrm{Cl}$ & $\mathrm{RR}$ & $95 \% \mathrm{Cl}$ & RR & $95 \% \mathrm{Cl}$ \\
\hline ALA & 0.94 & $0.76,1.17$ & 0.92 & $0.55,1.53$ & $0.91 \dagger$ & $0.70,1 \cdot 18$ & 0.58 & $0.25,1.36$ & 0.63 & $0.22,1.81$ & $0.60 \dagger$ & $0.30,1 \cdot 19$ \\
\hline$E P A+D H A$ & $0.60^{*}$ & $0.39,0.91$ & $0.59^{\star}$ & $0.40,0.89$ & $0.61^{\star \star} \dagger$ & $0.45,0.82$ & $0.55^{\star}$ & $0.31,0.96$ & $0.40^{* *}$ & $0.20,0.78$ & $0.50^{\star \star} \dagger$ & $0.31,0.79$ \\
\hline Total $n-3$ PUFA & $0.60^{*}$ & $0.39,0.91$ & $0.58^{\star \star}$ & $0.39,0.86$ & $0.60^{\star *} \dagger$ & $0.45,0.81$ & $0.53^{\star}$ & $0.30,0.92$ & $0.37^{\star \star}$ & $0.19,0.72$ & $0.49^{\star \star} \dagger$ & $0.30,0.75$ \\
\hline LA & 1.38 & $0.95,2.02$ & 1.08 & $0.78,1.50$ & $1.25 \dagger$ & $0.99,1.57$ & 1.43 & $0.83,2.46$ & $1 \cdot 16$ & $0.71,1.92$ & $1.35 \dagger$ & $0.93,1.97$ \\
\hline AA & $1.97^{\star *}$ & $1.21,3.21$ & 1.03 & $0.68,1.56$ & $1.35+\dagger$ & $0.99,1.84$ & 1.81 & $1.09,3.01$ & 1.02 & $0.55,1.89$ & $1.39 t$ & $0.90,2 \cdot 16$ \\
\hline Total $n-6$ PUFA & $1.77^{\star}$ & $1.07,2.94$ & 1.25 & $0.75,2.09$ & $1.54^{*} \dagger$ & $1 \cdot 09,2 \cdot 17$ & 1.42 & $0.84,2 \cdot 38$ & 1.64 & $0.87,3.07$ & $1.47 \dagger$ & $0.97,2.23$ \\
\hline Total $n-3: n-6$ ratio & $0.53^{\star \star}$ & $0.34,0.84$ & $0.55^{\star}$ & $0.34,0.88$ & $0.54^{\star \star \star} \dagger$ & $0.39,0.75$ & $0.49^{*}$ & $0.28,0.86$ & $0.34^{*}$ & $0.15,0.79$ & $0.43^{\star \star} \dagger$ & $0.26,0.70$ \\
\hline
\end{tabular}

AA, arachidonic acid (20:4n-6); ALA, $a$-linolenic acid (18:3n-3); DHA, $22: 6 n-3$; EPA, $20: 5 n-3$; LA, linoleic acid (18:2n-6); total $n-3$ ( $n-6)$ PUFA, the sum of $n$-3 (n-6) PUFA present in the chromatogram.

$P$ value for PUFA exposure-outcome association: * $P<0.05,{ }^{\star *} P<0.01,{ }^{* \star *} P<0.001$

$P$ value for PUFA exposure-cohort interaction: $\dagger P_{\text {for interaction }}>0.10, \dagger \dagger P_{\text {for interaction }}=0.06$.

† RR and $95 \% \mathrm{Cl}$ were calculated using generalised linear models for binary outcomes (modified Poisson) adjusted for maternal age, maternal BMI at study entry, gestational weight gain, maternal smoking during pregnancy, parity, parental education, parent atopy, child age at outcome assessment, child sex and for the pooled estimate a cohort indicator. Effect estimates correspond to a standard deviation score increase in PUFA and to a unit increase in the total $n-3: n-6$ ratio.

$\S$ Current wheeze was defined as the presence of wheezing or whistling in the chest in the past 12 months.

॥ Current asthma was defined as a positive answer to both ever-reported doctor diagnosis of asthma and the presence of wheezing or whistling in the chest in the past 12 months. 
PUFA at birth and allergy-related traits
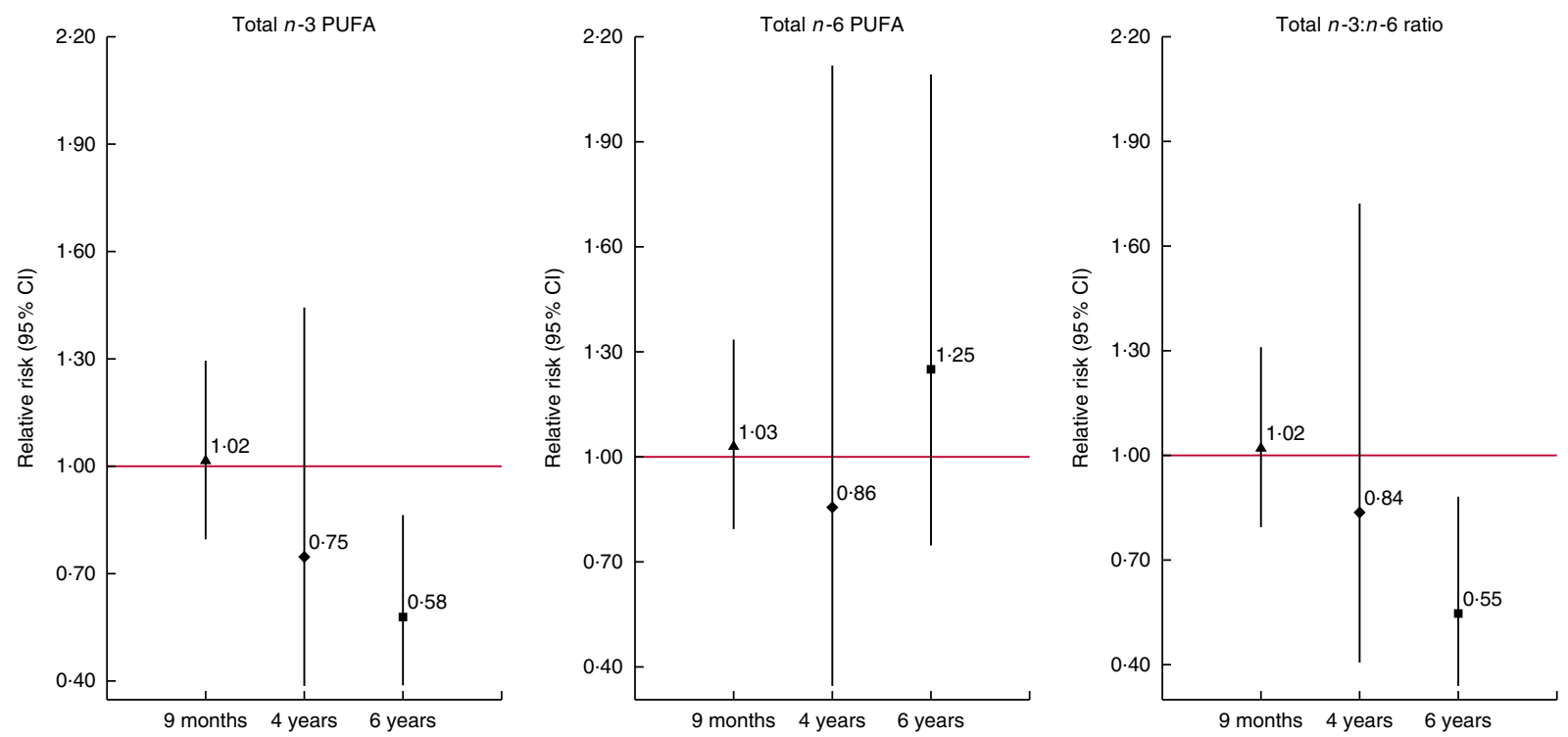

Fig. 1. Associations of cord blood phospholipid PUFA concentrations with current wheeze symptoms at 9 months, 4 years and 6 years of age in the RHEA Mother-Child cohort. Values are relative risks and their $95 \% \mathrm{Cl}$ calculated using generalised linear models for binary outcomes (modified Poisson). Models were adjusted for maternal age, maternal BMl at study entry, gestational weight gain, maternal smoking during pregnancy, parity, parental education, parent atopy, child age at outcome assessment and sex. Effect estimates correspond to a standard deviation score (SDS) increase in total $n$-3 ( $n-6)$ PUFA and to a unit increase in the total $n-3: n-6$ ratio. Current wheeze was defined as presence of wheezing or whistling in the chest since birth at 9 months of age ( $n / N$ 62/228) and in the past 12 months at 4 years $(n / N 10 / 222)$ and 6 years of age $(n / N 21 / 213)$. Total $n-3(n-6)$ PUFA, the sum of $n-3(n-6)$ PUFA present in the chromatogram. $\mathbf{\Delta}$, and $\square$, Effect estimate of each time point. Vertical bars represent $95 \%$ confidence intervals.

direction and size of the effect estimates (data not shown). In RHEA, results remained similar when we further adjusted for maternal Mediterranean diet adherence in pregnancy (data not shown). We found no associations with current wheeze symptoms in the other time points in RHEA, although a trend towards a reduction in wheeze risk with higher concentration of $n$-3 PUFA, and especially EPA + DHA, and a higher $n-3: n-6$ ratio was observed with increasing child age from 9 months to 6 years (Fig. 1 and online Supplementary Table S7).

\section{Sensitivity analyses}

The effect estimates did not materially change in complete case analysis or when additional adjustment was made for child BMI (data not shown). Effects estimates also remained similar after including birth weight and gestational age in multivariable models (online Supplementary Table S8). We found no evidence of effect measure modification by parent atopy for any of the outcomes of interest (online Supplementary Table S9). There was some evidence of effect measure modification by female sex on the multiplicative scale for the association between the n-3:n-6 PUFA ratio and current rhinitis symptoms (pooled RR $0.57 ; 95 \%$ CI $0.32,1.01 ; P=0.06$ ) (online Supplementary Table S10). However, no effect modification by child sex on either multiplicative or additive scale was observed for any of the other outcomes of interest (online Supplementary Table S10).

\section{Discussion}

In this analysis of prospective data of two birth cohorts from West and South Europe with different sociodemographic characteristics, we found that higher concentrations of $n-3$ PUFA, and especially of EPA and DHA, in cord blood were associated with reduced risk of current wheeze symptoms and asthma at 6-7 years of age. Higher cord blood $n-6$ PUFA concentrations were associated with a higher risk of current wheeze. We also observed a reduced risk of both current wheeze and asthma with increasing $n-3: n-6$ fatty acid ratio. The results were fairly homogeneous across the cohorts after controlling for a variety of confounders, and remained robust in the pooled and sensitivity analyses.

We measured cord blood phospholipid PUFA concentrations, which reflect recent maternal dietary intake at a time frame of 2-4 weeks ${ }^{(22)}$, as well as the level of fetal demand, and selectivity and efficiency of placental transfer ${ }^{(4)}$. Hence, they constitute good surrogates of fetal exposure in late pregnancy. The timing and biomarker used for assessing fetal exposure in our study are of high relevance as they coincide with the period during which there is an exponential increase in the rate of fetal fat accretion $^{(4)}$.

Fetal life is a critical time period of susceptibility during which nutritional exposures could have irreversible long-term influences on airway and immune function ${ }^{(44)}$. The $n-6$ and $n-3$ fatty acids have been proposed to exert immune-regulatory effects through involvement in several pathways. Animal and in vitro studies have shown that $n-6$ PUFA, as substrate for eicosanoid synthesis, can promote inflammation, favour T-helper cell type 2-driven immune responses and enhance the synthesis of Ig $\mathrm{E}$ antibodies $^{(6)}$. On the contrary, $n-3$ PUFA have been suggested to exert a protective effect by competing with the $n$ - 6 fatty acids for incorporation into cell membranes and for metabolism by enzymes of eicosanoid synthesis ${ }^{(6)}$. In addition, $n-3$ long-chain PUFA have been shown in vitro to alter the expression of inflammatory genes by modifying transcription factor activation, 
inhibit T-cell signalling through alteration of membrane lipid rafts, as well as to give rise to a family of anti-inflammatory mediators termed resolvins ${ }^{(45)}$.

Reports from human trials cannot unequivocally confirm or reject a beneficial effect of $n-3$ EPA and DHA supplementation in fetal life on allergy in childhood ${ }^{(7,8,46)}$. Findings from longitudinal cohort studies on prenatal PUFA exposure and childhood asthma or other allergy symptoms have also been mixed, with reports of null $^{(13,15,16)}$, beneficial (i.e. lower incidence) $)^{(11,12,17,47,48)}$ or even unfavourable $^{(14)}$ associations with higher $n$-3 PUFA or decreasing $n-6: n-3$ fatty acid ratio. Inconsistencies in findings may partly arise from exposure profile heterogeneity (i.e. categorisation and timing of exposure), and differences in outcome definition and adjustment for confounding variables. In the present study, we used two independent cohorts for replication, and we followed a centralised statistical analysis following a consensus protocol with harmonised information on exposure variables, potential confounders and child allergy-related symptoms. We found broadly similar associations across the different cohorts, although there was some evidence for between-cohort heterogeneity for the risk estimates of current wheeze in relation to $n-6 \mathrm{AA}$, for ever asthma in relation to $n-3$ ALA and for risk estimates of the $n-6$ GLA and DGLA. Such heterogeneity might have occurred owing to variability in the analytical methods used for cord blood fatty acid analysis and/or owing to differences in confounder patterns (e.g. differences in diet or lifestyle) between participating cohorts.

The EPA+DHA status in the Netherlands and Greece is characterised as very low-to-low, which is similar to most European and US populations following a Westernised diet $^{(23)}$. We found that $1 \mathrm{sD}$ increase in cord blood concentrations of $n$-3 PUFA, and especially of EPA and DHA, was associated with lower risk for childhood symptoms of wheeze and asthma. An inverse association with childhood wheeze and asthma risk was also observed for the $n-3: n-6$ PUFA ratio, which was driven to a great extent by $n$ - 3 long-chain PUFA. We did not have information on dietary intake in late pregnancy so as to disentangle the amount of dietary PUFA needed in order to achieve an SD change in cord blood long-chain PUFA concentrations. This change is feasible and likely reflects a significant dietary modification $^{(49)}$. Thus, a sizeable effect on wheeze and asthma symptoms could be achieved from dietary interventions leading to a marked increase in the $n-3: n-6$ PUFA ratio, and mainly in the intake of $n$ - 3 long-chain PUFA in late gestation. Indeed, in trials, a relation between fish-oil supplementation and effect becomes apparent at high doses, which are expected to significantly increase blood PUFA concentrations. In two trials conducted in Denmark, where population background levels of n-3 long-chain PUFA are higher compared with most European populations ${ }^{(23)}$, supplementation with high doses of fish oil in late gestation (providing $2.4 \mathrm{~g} \mathrm{EPA}+\mathrm{DHA} / \mathrm{d}$ from week 24 of pregnancy onwards ${ }^{(9)}$ or $2.7 \mathrm{~g} / \mathrm{d}$ from week 30 of pregnancy onwards $\left.{ }^{(50)}\right)$ resulted in significant increases $(>1 \mathrm{sD})$ in blood $n$-3 long-chain PUFA concentrations and lower risk of offspring wheeze and asthma compared with placebo. In terms of safety, high doses of $n$ - 3 long-chain PUFA up to $2.7 \mathrm{~g} / \mathrm{d}$ in pregnancy through fish-oil supplementation have not been associated with serious adverse effects, such as bleeding complications $^{(51)}$.
Our findings are partly in line with the initial Black and Sharpe hypothesis, suggesting that the balance between $n-3$ and $n-6$ PUFA might have implications in the development of asthma and other allergy-related symptoms ${ }^{(3)}$. Despite the unifying hypotheses that have been developed to explain the common co-existence of allergic disease symptoms in a child $^{(52)}$, asthma, rhinitis and eczema represent different disease entities with different underlying mechanisms ${ }^{(53-55)}$. In the present study, we observed associations only for symptoms of wheeze and asthma, suggesting a specific effect of prenatal PUFA on airway development and function. Further observational and experimental studies using spirometry and markers of airway inflammation are needed for replication and to explore the long-term effects of prenatal PUFA exposure.

A recent systematic review highlighted the importance of planning a subgroup analysis by sex in studies assessing the health effects of perinatal PUFA ${ }^{(56)}$. Sexual dimorphism in fatty acid metabolism has been suggested to exist owing to sex differences in rates of $\beta$-oxidation and fat mobilisation, and possible influences of sex hormones on the enzymatic synthesis of long-chain fatty acids ${ }^{(56,57)}$. A previous analysis in the Generation R reported no effect measure modification in the association between maternal PUFA concentrations with childhood atopic diseases by offspring $\operatorname{sex}^{(13)}$. In our study, we found some evidence for effect measure modification by female sex on the multiplicative scale for the association between the ratio of total $n-3: n-6$ PUFA at birth with rhinitis symptoms in childhood. However, we did not observe any effect modification by sex for the other outcomes of interest. Thus, we treat this result with caution, as we cannot rule out the possibility that the observed effect modification for rhinitis is due to chance.

As in all observational studies, there is the possibility of unmeasured residual confounding. Although, in our analyses, we took into account a variety of maternal and child lifestyle characteristics, including Mediterranean diet adherence during pregnancy $^{(32)}$ and child biomarker levels of PUFA, we cannot exclude the possibility that other differences in diet or lifestyle factors might have influenced the observed findings. However, we did not see strong evidence for sociodemographic confounding in our analyses. Similar to most cohort studies, a limitation of the present study is attrition. It is possible that loss to follow-up could have introduced bias. Nevertheless, many sociodemographic characteristics and the cord blood PUFA concentrations were similar in the study sample and in those excluded. Child allergic-disease symptoms were ascertained by symptom-based questionnaires, which could lead to outcome misclassification. However, both participating cohorts used questionnaires adapted from the ISAAC study ${ }^{(28)}$. Moreover, to enhance asthma and eczema outcome accuracy, we used composite definitions that require many conditions to be met. Although assessment of several PUFA exposures and child outcomes might raise concern about multiple testing, in our opinion an application of correction for multiple comparisons might be inappropriate in this case given the high correlations among exposures and among outcomes ${ }^{(58)}$.

In this pooled analysis of two cohorts from West and South Europe, we found that high concentrations of $n$ - 3 PUFA, particularly EPA and DHA, and a high $n-3: n-6$ ratio in cord blood 
were associated with reduced risk of current wheeze and asthma in mid-childhood. Our findings suggest that dietary interventions resulting in a marked increase in the dietary $n-3: n-6$ PUFA ratio, and mainly in the intake of $n-3$ long-chain PUFA in late gestation are likely to reduce the risk of asthma symptoms in mid-childhood.

\section{Acknowledgements}

The authors would like to thank Professor Emeritus Gerard Hornstra, who made the MEFAB study possible. The authors are also extremely grateful to the participants of the MEFAB and RHEA cohorts for their time and commitment.

MEFAB: The MEFAB cohort was financially supported by the Dutch Organization for Scientific Research (NWO, grant no. 904 62 186), and the University Hospital of Maastricht (Profilerings Fonds).

RHEA: The Mother-Child Cohort in Crete project was financially supported by European projects (EU FP6-003-Food3-NewGeneris - contract no. 16320, EU FP6 STREP Hiwatecontract no. 36224, EU FP7 ENV.2007.1.2.2.2. Project No. 211250 Escape, EU FP7-2008-ENV-1.2.1.4 Envirogenomarkers contract no. 226756, EU FP7-HEALTH-2009-single stage CHICOS contract no. 241604, EU FP7 ENV.2008.1.2.1.6. proposal no. 226285 ENRIECO, EU-FP7, proposal no. 264357 MeDALL, EU- FP7- HEALTH-2012 proposal no. 308333 HELIX), and the Greek Ministry of Health (Program of Prevention of obesity and neurodevelopmental disorders in preschool children, in Heraklion district, Crete, Greece: 2011-2014; 'Rhea Plus': Prevention Program of Environmental Risk Factors for Reproductive Health, and Child Health: 2012-2015.

N. S., M. G., L. C. and M. P. Z. conceived the study and had primary responsibility for the final content; M. A. and E. G. S. conducted the assay of fatty acids for the RHEA cohort; all authors were involved in the design and planning of the study, and the data collection; N. S. and K. M. analysed data; N. S., M. G. and L. C. wrote the paper; and all authors critically reviewed and approved the final manuscript.

None of the authors has any conflicts of interest to declare.

\section{Supplementary material}

For supplementary material/s referred to in this article, please visit https://doi.org/10.1017/S0007114517003348

\section{References}

1. Asher MI, Montefort S, Bjorksten B, et al. (2006) Worldwide time trends in the prevalence of symptoms of asthma, allergic rhinoconjunctivitis, and eczema in childhood: ISAAC Phases One and Three repeat multicountry cross-sectional surveys. Lancet 368, 733-743.

2. Devereux G (2006) The increase in the prevalence of asthma and allergy: food for thought. Nat Rev Immunol 6, 869-874.

3. Black PN \& Sharpe S (1997) Dietary fat and asthma: is there a connection? Eur Respir J 10, 6-12.

4. Haggarty P (2010) Fatty acid supply to the human fetus. Annu Rev Nutr 30, 237-255.
5. Prescott SL \& Dunstan JA (2007) Prenatal fatty acid status and immune development: the pathways and the evidence. Lipids 42, 801-810.

6. Calder PC, Kremmyda LS, Vlachava M, et al. (2010) Is there a role for fatty acids in early life programming of the immune system? Proc Nutr Soc 69, 373-380.

7. Best KP, Gold M, Kennedy D, et al. (2016) Omega-3 long-chain PUFA intake during pregnancy and allergic disease outcomes in the offspring: a systematic review and metaanalysis of observational studies and randomized controlled trials. Am J Clin Nutr 103, 128-143.

8. Best KP, Sullivan T, Palmer D, et al. (2016) Prenatal fish oil supplementation and allergy: 6-year follow-up of a randomized controlled trial. Pediatrics 137, e20154443.

9. Bisgaard H, Stokholm J, Chawes BL, et al. (2016) Fish oilderived fatty acids in pregnancy and wheeze and asthma in offspring. $N$ Engl J Med 375, 2530-2539.

10. Hornstra $G$ (2000) Essential fatty acids in mothers and their neonates. Am J Clin Nutr 71, 1262S-1269S.

11. Pike KC, Calder PC, Inskip HM, et al. (2012) Maternal plasma phosphatidylcholine fatty acids and atopy and wheeze in the offspring at age of 6 years. Clin Dev Immunol 2012, 474613 .

12. Newson RB, Shaheen SO, Henderson AJ, et al. (2004) Umbilical cord and maternal blood red cell fatty acids and early childhood wheezing and eczema. J Allergy Clin Immunol 114, 531-537.

13. Rucci E, den Dekker HT, de Jongste JC, et al. (2016) Maternal fatty acid levels during pregnancy, childhood lung function and atopic diseases. The Generation R Study. Clin Exp Allergy 46, 461-471.

14. Notenboom ML, Mommers M, Jansen EH, et al. (2011) Maternal fatty acid status in pregnancy and childhood atopic manifestations: KOALA Birth Cohort Study. Clin Exp Allergy 41, 407-416.

15. Standl M, Demmelmair H, Koletzko B, et al. (2014) Cord blood LC-PUFA composition and allergic diseases during the first 10 yr. Results from the LISAplus study. Pediatr Allergy Immunol 25, 344-350.

16. Yu YM, Chan YH, Calder PC, et al. (2015) Maternal PUFA status and offspring allergic diseases up to the age of 18 months. Br J Nutr 113, 975-983.

17. Montes R, Chisaguano AM, Castellote AI, et al. (2013) Fattyacid composition of maternal and umbilical cord plasma and early childhood atopic eczema in a Spanish cohort. Eur J Clin Nutr 67, 658-663.

18. van der Wurff ISM, de Groot RHM, Stratakis N, et al. (2015) Maastricht essential fatty acid birth cohort. Lipid Technol 27, 59-62.

19. Chatzi L, Plana E, Daraki V, et al. (2009) Metabolic syndrome in early pregnancy and risk of preterm birth. Am J Epidemiol 170, 829-836.

20. Al MDM, Vanhouwelingen AC, Kester ADM, et al. (1995) Maternal essential fatty-acid patterns during normalpregnancy and their relationship to the neonatal essential fatty-acid status. Br J Nutr 74, 55-68.

21. Glaser C, Demmelmair H \& Koletzko B (2010) Highthroughput analysis of total plasma fatty acid composition with direct in situ transesterification. PLOS ONE 5, e12045.

22. Hodson L, Skeaff CM \& Fielding BA (2008) Fatty acid composition of adipose tissue and blood in humans and its use as a biomarker of dietary intake. Prog Lipid Res 47, 348-380.

23. Stark KD, Van Elswyk ME, Higgins MR, et al. (2016) Global survey of the omega-3 fatty acids, docosahexaenoic acid and eicosapentaenoic acid in the blood stream of healthy adults. Prog Lipid Res 63, 132-152. 
24. Calder PC (2012) Mechanisms of Action of (n-3) fatty acids. J Nutr 142, 592s-599s.

25. Schmitz G \& Ecker J (2008) The opposing effects of $n-3$ and n-6 fatty acids. Prog Lipid Res 47, 147-155.

26. Hornstra G (2005) Essential fatty acids during pregnancy. Impact on mother and child. Nestle Nutr Workshop Ser Pediatr Program 55, 83-96; discussion 96-100.

27. de Vries PS, Gielen M, Rizopoulos D, et al. (2014) Association between polyunsaturated fatty acid concentrations in maternal plasma phospholipids during pregnancy and offspring adiposity at age 7: the MEFAB cohort. Prostaglandins Leukot Essent Fatty Acids 91, 81-85.

28. Asher MI, Keil U, Anderson HR, et al. (1995) International Study of Asthma and Allergies in Childhood (ISAAC): rationale and methods. Eur Respir J 8, 483-491.

29. Pearce N, Ait-Khaled N, Beasley R, et al. (2007) Worldwide trends in the prevalence of asthma symptoms: phase III of the International Study of Asthma and Allergies in Childhood (ISAAC). Thorax 62, 758-766.

30. Bjorksten B, Clayton T, Ellwood P, et al. (2008) Worldwide time trends for symptoms of rhinitis and conjunctivitis: Phase III of the International Study of Asthma and Allergies in Childhood. Pediatr Allergy Immunol 19, 110-124.

31. Pinart M, Benet M, Annesi-Maesano I, et al. (2014) Comorbidity of eczema, rhinitis, and asthma in IgE-sensitised and non-IgE-sensitised children in MeDALL: a population-based cohort study. Lancet Respir Med 2, 131-140.

32. Chatzi L, Torrent M, Romieu I, et al. (2008) Mediterranean diet in pregnancy is protective for wheeze and atopy in childhood. Thorax 63, 507-513.

33. Rump P, Popp-Snijders C, Heine RJ, et al. (2002) Components of the insulin resistance syndrome in seven-year-old children: relations with birth weight and the polyunsaturated fatty acid content of umbilical cord plasma phospholipids. Diabetologia 45, 349-355.

34. Zou G (2004) A modified Poisson regression approach to prospective studies with binary data. Am J Epidemiol 159, 702-706.

35. Ben J (2008) NLCHECK: Stata Module to Check Linearity Assumption After Model Estimation. Statistical Software Components S456968. Boston, MA: Boston College Department of Economics.

36. Rubin DB (2004) Multiple Imputation for Nonresponse in Surveys. Hoboken, NJ: Wiley-Interscience.

37. White IR, Royston P \& Wood AM (2011) Multiple imputation using chained equations: Issues and guidance for practice. Stat Med 30, 377-399.

38. Almqvist C, Worm M, Leynaert B, et al. (2008) Impact of gender on asthma in childhood and adolescence: a GA2LEN review. Allergy 63, 47-57.

39. Nurmatov U, Nwaru BI, Devereux G, et al. (2012) Confounding and effect modification in studies of diet and childhood asthma and allergies. Allergy 67, 1041-1059.

40. Duchen K \& Bjorksten B (2001) Polyunsaturated n-3 fatty acids and the development of atopic disease. Lipids $\mathbf{3 6}$, $1033-1042$.
41. Knol MJ, van der Tweel I, Grobbee DE, et al. (2007) Estimating interaction on an additive scale between continuous determinants in a logistic regression model. Int $J$ Epidemiol 36, 1111-1118.

42. VanderWeele TJ \& Knol MJ (2014) A tutorial on interaction. Epidemiol Methods 3, 33-72.

43. Faul F, Erdfelder E, Buchner A, et al. (2009) Statistical power analyses using G*Power 3.1: tests for correlation and regression analyses. Behav Res Methods 41, 1149-1160.

44. Devereux G (2007) Early life events in asthma-diet. Pediatr Pulmonol 42, 663-673.

45. Calder PC, Krauss-Etschmann S, de Jong EC, et al. (2006) Early nutrition and immunity - progress and perspectives. Br J Nutr 96, 774-790.

46. Ramaswami R, Serhan CN, Levy BD, et al. (2016) Fish oil supplementation in pregnancy. N Engl J Med 375, 2599-2601.

47. Lumia M, Luukkainen P, Tapanainen H, et al. (2011) Dietary fatty acid composition during pregnancy and the risk of asthma in the offspring. Pediatr Allergy Immunol 22, 827-835.

48. Miyake Y, Sasaki S, Tanaka K, et al. (2009) Maternal fat consumption during pregnancy and risk of wheeze and eczema in Japanese infants aged 16-24 months: the Osaka Maternal and Child Health Study. Thorax 64, 815-821.

49. Miles EA, Noakes PS, Kremmyda LS, et al. (2011) The Salmon in Pregnancy Study: study design, subject characteristics, maternal fish and marine $n-3$ fatty acid intake, and marine $n-3$ fatty acid status in maternal and umbilical cord blood. Am J Clin Nutr 94, 1986S-1992S.

50. Olsen SF, Osterdal ML, Salvig JD, et al. (2008) Fish oil intake compared with olive oil intake in late pregnancy and asthma in the offspring: 16 y of registry-based follow-up from a randomized controlled trial. Am J Clin Nutr 88, 167-175.

51. Makrides M, Duley L \& Olsen SF (2006) Marine oil, and other prostaglandin precursor, supplementation for pregnancy uncomplicated by pre-eclampsia or intrauterine growth restriction. Cochrane Database Syst Rev, issue 3, CD003402.

52. Spergel JM \& Paller AS (2003) Atopic dermatitis and the atopic march. J Allergy Clin Immunol 112, Suppl. 6, S118-S127.

53. Martinez FD \& Vercelli D (2013) Asthma. Lancet 382, 1360-1372.

54. Bieber T (2008) Atopic dermatitis. $N$ Engl J Med 358, $1483-1494$.

55. Skoner DP (2001) Allergic rhinitis: definition, epidemiology, pathophysiology, detection, and diagnosis. J Allergy Clin Immunol 108, Suppl. 1, S2-S8.

56. Decsi T \& Kennedy K (2011) Sex-specific differences in essential fatty acid metabolism. Am J Clin Nutr 94, Suppl. 6, S1914-S1919.

57. Childs CE, Romeu-Nadal M, Burdge GC, et al. (2008) Gender differences in the $n-3$ fatty acid content of tissues. Proc Nutr Soc 67, 19-27.

58. Rothman KJ (1990) No adjustments are needed for multiple comparisons. Epidemiology 1, 43-46. 